\title{
Etude d'un accident en fromagerie de type «Camembert" causé par des mucorales
}

\author{
par \\ M. BRENET, J. L. CENTELEGHE, J. B. MILLIERE, \\ J. P. RAMET et F. WEBER \\ Laboratoire de Technologie, Ecole Supérieure de Laiterie de Nancy
}

\section{I. - Introduction}

Pendant la période hivernale et dans plusieurs usines de l'Est de la France, divers accidents d'origine mycologique, affectant la présentation des fromages à pâte molle de type Camembert, ont attiré notre attention.

Les praticiens confondent cet accident avec celui du « Poil de chat » dont l'agent habituel est Mucor mucedo Brefeld.

Quatre à cinq jours après la fabrication des fromages, des touffes noirâtres de moisissures apparaissent sur la croûte et persistent jusqu'à l'emballage du produit. Leur répartition semble être le fait du hasard, mais elles sont souvent en plus grand nombre sur l'une des deux faces. Un examen plus minutieux montre que généralement la moisissure est localisée dans des zones rosées de la croûte, où le Penicillium caseicolum pousse très difficilement.

\section{II. - Détermination de la moisissure responsable de l'accident}

Plusieurs moisissures appartenant à l'ordre des Mucorales; ont été isolées sur les fromages contaminés. Une espèce est largement dominante : Mucor globosus Fischer.

Cultivée en boîte de Pétri, sur gélose au malt, cette moisissure forme à $20^{\circ} \mathrm{C}$ des colonies rondes, envahissantes, formées au départ d'un mycélium blanc qui prend des teintes grises en vieillissant. Les colonies montrent deux parties distinctes. Le centre plus épais, plus grisâtre porte les fructifications : des petits sporanges brun foncé se dressent à plus d'un centimètre au-dessus du mycélium ; le pourtour de la colonie est formé d'un mycélium rayonnant, plus ténu qui ne porte pas de fructifications. 
$\mathrm{Au}$ microscope, la moisissure montre un mycélium hyalin non cloisonné, d'où partent des sporangiophores hyalins qui portent des sporanges brun foncé. Les sporangiophores (de 10 à $20 \mu$ de diamètre), sont longs ( 1 à $2 \mathrm{~cm}$ ), non cloisonnés et sans chlamydospores.

Les sporanges (100 $\mu$ de diamètre) sont ronds et formés d'une centaine de spores, rondes à maturité ( $5 \mu$ de diamètre), agglutinées par un mucus autour d'une columelle nue, ovale (parfois pyriforme) qui mesure de 40 à $50 \mu$ de long. La paroi des sporanges est fine et elle disparaît à maturité.

Le mycélium des colonies âgées possède des chlamydospores et il est souvent cloisonné en grands articles.

Ces caractères permettent d'identifier un Mucor globosus Fischer, de la classe des Phycomycètes, ordre des Mucorales, famille des Mucoraceae.

\section{III. - Aperçu de la biologie des Mucorales}

Ces moisissures sont très abondantes dans la nature où elles se développent sur des substrats organiques divers. Elles sont incapables d'assurer la dégradation de molécules organiques complexes : lignine, cellulose. Elles ne peuvent se développer qu'aux dépens de composés carbonés simples tels les sucres solubles. Ce sont donc des saprophytes primaires qui épuisent rapidement le milieu colonisé.

Leurs handicaps traditionnels sont compensés par une croissance végétative rapide, et par un cycle très court. Les spores émises en très grand nombre germent rapidement, lorsqu'elles trouvent un substrat favorable. Ce sont des agents colonisateurs dangereux : leurs colonies luxuriantes étouffent parfois le thalle d'autres espèces à croissance plus lente.

Contrairement aux Penicillia qui possèdent des xérospores qui se détachent du thalle au moindre mouvement d'air, les Mucorales forment des myxospores agglutinées dans un mucus dont la dispersion se fait par l'eau. Les locaux aux parois humides et à atmosphère saturée sont très aptes à leur dissémination. Si un sporange tombe sur un fromage en cours d'égouttage, les spores sont dispersées et vont contaminer les autres fromages grâce au sérum qui s'écoule.

Des sporanges entiers peuvent être véhiculés par le vent ; mais leur dissémination se fait surtout par les insectes, les animaux et les vêtements auxquels ils adhèrent.

\section{IV. - Caractères biochimiques du mucor globosus}

Nous avons comparé la croissance du Mucor globosus à celle du Penicillium caseicolum employé dans les usines où nous fûmes appelés, en fonction de la température et du $\mathrm{pH}$. 


\section{TABLEAU 1}

Croissance comparée de Mucor globosus et des souches de Penicillium caseicolum en fonction de la température et du temps (Les chiffres représentent les diamètres moyens des colonies en millimètres)

\begin{tabular}{|c|c|c|c|c|c|c|c|c|c|c|}
\hline Température & \multicolumn{2}{|c|}{$4^{\circ} \mathrm{C}$} & \multicolumn{2}{|c|}{$13^{\circ} \mathrm{C}$} & \multicolumn{2}{|c|}{$17^{\circ} \mathrm{C}$} & \multicolumn{2}{|c|}{$23^{\circ} \mathrm{C}$} & \multicolumn{2}{|c|}{$29^{\circ} \mathrm{C}$} \\
\hline Espèce & M.g. & P.c. & M.g. & P.c. & M.g. & P.c. & M.g. & P.c. & M.g. & P.c. \\
\hline $22 \mathrm{~h}$ & 0 & 0 & 0 & 0 & 3 & 0 & 7 & 0 & 0 & 0 \\
\hline $47 \mathrm{~h}$ & 0 & 0 & 8 & 0 & 15 & 0,5 & 24 & 2 & 0 & 0 \\
\hline $78 \mathrm{~h}$ & 0 & 0 & 20 & 0 & 30 & 7 & 47 & 9 & 0 & 0 \\
\hline $6 j$ & 0 & 0 & 52 & 12 & 80 & 20 & 90 & 24 & 0 & 0 \\
\hline
\end{tabular}


Croissance comparée de Mucor globosus et des souches de Penicillium caseicolum en fonction du $\mathrm{pH}$ et du temps (Les nombres représentent les diamètres moyens des colonies en millimètres)

\begin{tabular}{|c|c|c|c|c|c|}
\hline $\mathrm{pH}$ & & $24 \mathrm{~h}$ & $48 \mathrm{~h}$ & $72 \mathrm{~h}$ & $6 j$ \\
\hline 3 & $\begin{array}{l}\text { M.g. } \\
\text { P.c. }\end{array}$ & $\begin{array}{l}0 \\
0\end{array}$ & $\begin{array}{l}5 \\
1\end{array}$ & $\begin{array}{l}9 \\
5\end{array}$ & $\begin{array}{l}22 \\
19\end{array}$ \\
\hline 3,5 & $\begin{array}{l}\text { M.g. } \\
\text { P.c. }\end{array}$ & $\begin{array}{l}4 \\
0\end{array}$ & $\begin{array}{r}16 \\
2\end{array}$ & $\begin{array}{r}30 \\
6\end{array}$ & $\begin{array}{l}75 \\
20\end{array}$ \\
\hline 4 & $\begin{array}{l}\text { M.g. } \\
\text { P.c. }\end{array}$ & $\begin{array}{l}6 \\
0\end{array}$ & $\begin{array}{r}23 \\
2\end{array}$ & $\begin{array}{r}45 \\
7\end{array}$ & ${ }^{*}$ \\
\hline 5,1 & $\begin{array}{l}\text { M.g. } \\
\text { P.c. }\end{array}$ & $\begin{array}{l}7 \\
0\end{array}$ & $\begin{array}{r}24 \\
2\end{array}$ & $\begin{array}{r}47 \\
7\end{array}$ & $\stackrel{*}{24}$ \\
\hline 5,9 & $\begin{array}{l}\text { M.g. } \\
\text { P.c. }\end{array}$ & $\begin{array}{l}7 \\
0\end{array}$ & $\begin{array}{r}27 \\
2\end{array}$ & $\begin{array}{r}50 \\
8\end{array}$ & $\begin{array}{c}* \\
27\end{array}$ \\
\hline 6,8 & $\begin{array}{l}\text { M.g. } \\
\text { P.c. }\end{array}$ & $\begin{array}{l}9 \\
0\end{array}$ & $\begin{array}{r}28 \\
3\end{array}$ & $\begin{array}{r}52 \\
7\end{array}$ & * 26 \\
\hline 7,2 & $\begin{array}{l}\text { M.g. } \\
\text { P.c. }\end{array}$ & $\begin{array}{l}5 \\
0\end{array}$ & $\begin{array}{r}24 \\
3\end{array}$ & $\begin{array}{r}43 \\
7\end{array}$ & $\stackrel{*}{*}$ \\
\hline 8,9 & $\begin{array}{l}\text { M.g. } \\
\text { P.c. }\end{array}$ & $\begin{array}{l}3 \\
0\end{array}$ & $\begin{array}{r}21 \\
2\end{array}$ & $\begin{array}{r}39 \\
6\end{array}$ & * 25 \\
\hline
\end{tabular}

* : boîte de $90 \mathrm{~mm}$ de diamètre entièrement colonisée. 
L'étude de l'action de la température est effectuée sur un milieu gélosé au malt ajusté à $\mathrm{pH} 5,1$. Celle de l'influence du $\mathrm{pH}$ est réalisée sur ce même milieu amené à différentes valeurs par adjonction, après autoclavage, de soude ou d'acide lactique stérile.

Les milieux sont coulés en boîte de Pétri et séchés $2 \mathrm{~h}$ à l'étuve à $30^{\circ} \mathrm{C}$. L'ensemencement se fait par une piqûre centrale apportant le même nombre de spores pour les deux espèces. La croissance est estimée par le diamère en millimètres des colonies. Les résultats sont consignés dans les tableaux 1 et 2.

L'étude de la croissance en fonction de la température confirme la luxuriance du Mucor globosus et sa rapidité de croissance à $23^{\circ} \mathrm{C}$. Par contre on ne note aucun développement pour les deux espèces à $4^{\circ} \mathrm{C}$ et à $29^{\circ} \mathrm{C}$.

Le Mucor globosus se développe dans une large gamme de $\mathrm{pH}$. Son optimum semble se situer de $\mathrm{pH} 4$ à $\mathrm{pH} 7,2$ avec un maximum pour des $\mathrm{pH}$ de 6 à 7. Les souches de Penicillium caseicolum employées couvrent une trop large gamme de $\mathrm{pH}$; nous pensons qu'il faudrait leur incorporer des variétés se développant rapidement de $\mathrm{pH} 4,5$ à 5 .

Le Mucor globosus cultivé sur un milieu à la caséine ne développe pas de colonies. Il semble donc qu'il ne possède pas l'équipement enzymatique suffisant pour dégrader ce substrat. Par contre, quand il se développe aux dépens d'azote sous forme d'ions $\mathrm{NO}_{3}-$, d'ions $\mathrm{NH}_{4}+$ ou de polypeptides sa croissance est excessivement luxuriante. Sur des milieux contenant de l'azote sous forme de polypeptides, nous avons remarqué qu'au bout de $48 \mathrm{~h}$, les colonies de $25 \mathrm{~mm}$ de diamètre, portaient déjà des sporanges. Donc tous les facteurs qui augmentent la fraction d'azote soluble dans le lait permettent le développement de la moisissure.

Nous avons de plus mis en évidence une légère baisse de la croissance du Mucor globosus sur des milieux contenant 3 p. 100 de $\mathrm{NaCl}$, mais aux taux inférieurs employés en fromagerie ce produit ne constitue pas un moyen de lutte efficace.

\section{V. - Modalités de la contamination}

Pour déterminer le début de la contamination, nous avons utilisé une méthode dite d' "empreinte ». Dans des boîtes de Pétri stériles en verre, d'un diamètre de $150 \mathrm{~mm}$, on coule $40 \mathrm{~cm}^{3}$ de gélose au malt. Après séchage à l'étuve, on applique la face d'un fromage à la surface du milieu. Au cours de l'incubation, on suit le développement des moisissures.

Par cette méthode, nous avons suivi différentes fabrications et nous avons remarqué que les fromages étaient contaminés dès le 
moulage. Par contre dans le lait emprésuré, nous n'avons pas mis de Mucorales en évidence.

Il semble donc que la contamination ait lieu dès la mise en moules. Pendant toute la durée de l'égouttage, les spores peuvent germer et développer leurs hyphes mycéliennes, car la température de la salle $\left(24\right.$ à $27^{\circ} \mathrm{C}$ ) leur convient fort bien. Elles trouvent dans le lactosérum surnageant des substances solubles facilement assimilables qui permettent leur germination, même dans des conditions d'anaérobiose.

La lecture des boîtes d'empreintes a montré que la première face en contact avec le store est généralement la plus contaminée. Avant leur utilisation, les plateaux garnis de stores et de moules sont souvent stockés dans les salles de fabrication. Il se produit alors une contamination du matériel et notamment des stores par les myxospores présentes dans l'ambiance humide de la salle ou dans les gouttes de condensation qui se forment au contact des parois froides.

Sur des fromages âgés de 3 à $4 \mathrm{j}$, nous avons remarqué que le Mucor globosus pousse sur des zones rosées de la croûte. Ces zones montrent une grande prolifération des levures et de Geotrichum $s p$. qui comme les Mucorales possèdent des myxospores. Il semble donc que ces germes soient apportés dans les mêmes conditions. Il se peut même que le Mucor globosus utilise les produits de dégradation de ces micro-organismes, pour assurer sa croissance.

\section{VI. - Remèdes proposés}

Des études d'atmosphère et des grattages de parois ont montré que les germes contaminants étaient très solidement implantés dans les usines. En premier lieu, nous proposerons donc des mesures d'hygiène des locaux, et en deuxième lieu nous envisagerons le facteur fromage.

Nous avons déjà insisté sur l'importance des eaux de condensation dans la dissémination des spores de Mucorales. Aussi n'est-il pas surprenant que ces moisissures se manifestent surtout en hiver. Une bonne précaution est de nettoyer soigneusement et de désinfecter les murs et les sols des salles de fabrication, et de peindre plafonds et murs avec des peintures antifongiques. Il faut se méfier des sol usagés qui sont poreux, et des bouches d'égout. Pour la désinfection des parois, des solutions à base d'iode ont donné de bons résultats. Il faut de plus éviter les condensations et baisser l'humidité relative de l'air en ventilant les salles. Toutefois après chaque désinfection, il faut pulvériser une suspension de spores de Penicillium caseicolum.

Dans les usines, nous avons remarqué que la moisissure apparaît au départ sur des fromages à caractère présure très marqué (type 
" pâte stabilisée ») et que avec le temps, elle gagne les autres variétés de fromages pour atteindre en dernier lieu des fromages de type Camembert. L'acidité des produits ne peut pas être mis en cause, puisque pour toutes les variétés elle oscille au démoulage entre $\mathrm{pH} 4,7$ et $\mathrm{pH} 4,9$. Au contraire, la vitesse d'acidification est un facteur primordial sur lequel on peut jouer. Une acidification rapide en début de fabrication donne un fromage bien égoutté. Sa surface est rapidement ressuyée et les myxospores germent mal, car elles ne trouvent pas les substances solubles et l'eau nécessaire. Dans le même ordre d'idée, un ressuyage efficace doit être réalisé après le saumurage des fromages.

La croissance du Mucor globosus est luxuriante quand il dégrade des polypeptides. A ce sujet, il est bon de noter que la phase tertiaire de protéolyse générale due à l'action de la présure commence dès l'emprésurage. Au moment du moulage, la proportion de caséine dégradée est déjà notable. De plus cette protéolyse dépend de la concentration en enzyme. Le stockage du lait augmente la proportion de caséine dégradée. Dans une usine qui utilise du lait stocké pendant 4 à $5 \mathrm{j}$ à $4^{\circ} \mathrm{C}$, nous avons trouvé $13 \mathrm{~g}$ d'azote soluble et $4,3 \mathrm{~g}$ de NPN par litre de lait. Ces dégradations sont dues à une abondante flore de bactéries psychrophiles caséolytiques $\left(2 \times 10^{6}\right.$ germes par $\mathrm{ml}$ ).

\section{Conclusion}

La contamination commence dès le moulage des fromages, et le Mucor globosus se développe préférentiellement sur des zones rosées de la croûte où prolifèrent un grand nombre de Geotrichum sp. et de levures. Le Penicillium caseicolum ensemencé dans le lait pousse très faiblement à ces endroits. Nous pensons que les Geotrichum sp. et les levures forment une couche imperméable à l'oxygène et qu'à cause de ces conditions d'anaérobiose partielle, le Penicillium caseicolum ne peut pas germer. Des essais ont montré que sur des fromages pulvérisés avec une suspension de spores, l'accident est beaucoup moins spectaculaire.

L'eau joue un rôle très important dans la dissémination des spores de Mucorales. Pour lutter contre ces moisissures, il faut briser leur cycle dans l'usine, particulièrement au niveau de la salle de fabrication (laver et désinfecter murs, sols et plafonds, et baisser l'humidité relative de l'air).

Un long stockage du lait, en augmentant la proportion de caséine dégradée, permet une bonne implantation de Mucor globosus sur les fromages. De même la croissance de cette moisissure est plus importante sur des fromages dont la vitesse d'acidification est faible en début d'égouttage. 


\section{S u m m a ry}

The authors study an accident coming from moulds, in winter, in cheese factories in the East of France, specialized in cheese making camemberts.

They identify the responsible species Neurospora sitophila Saccardo and especially Mucor globosus Fischer ; they study the principal biochemical properties of this latter.

\section{Bibliographie}

BRENET (M.) (1971). - D.E.A. d'Agronomie.

Gilman (J. C) (1957). - A manual of soil Fungi. The Iowa State University Press. JACQuet (J.) et Desfleurs (M.) (1966). - Etude d'un nouvel accident de "bleu » en fromagerie de camembert. Enseignements pratiques.

MAsson (A.) (1959). - Contribution à l'étude de l'amélioration des sels de fromagerie. Cas du salage à $\sec$ des fromages à pâte molle et à croûte moisie. Bull. Soc. Sci., Nancy, 18, p. 2-69.

RaPilly (F.) (1968). - Les techniques de mycologie en pathologie végétale. Annales des épiphyties.

Sмiтh (G.) (1959). - An introduction to industrial mycology. Edward Arnold ldt. 